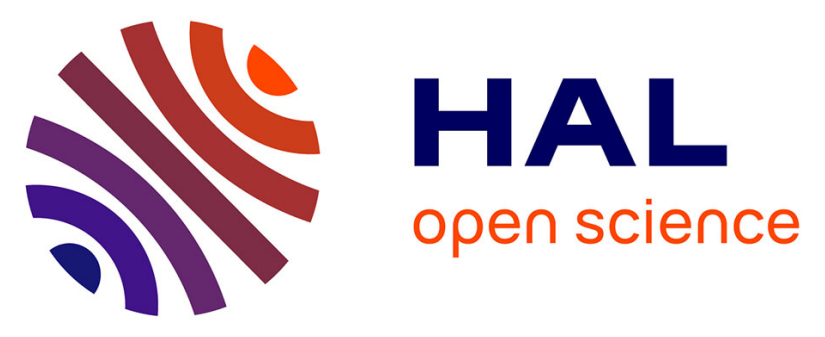

\title{
Transport mechanisms in 8-tris-hydroxyquinoline aluminium (Alq3) electronic layers: a study by photodipolar absorption
}

André Moliton, Bruno Lucas, Sebastien Berthon, Wassim Rammal, Roger

Clive Hiorns

\section{To cite this version:}

André Moliton, Bruno Lucas, Sebastien Berthon, Wassim Rammal, Roger Clive Hiorns. Transport mechanisms in 8-tris-hydroxyquinoline aluminium (Alq3) electronic layers: a study by photodipolar absorption. Philosophical Magazine, 2006, 86 (19), pp.2847-2863. 10.1080/14786430600636351 . hal01003839

\section{HAL Id: hal-01003839 \\ https://hal.science/hal-01003839}

Submitted on 10 Jun 2014

HAL is a multi-disciplinary open access archive for the deposit and dissemination of scientific research documents, whether they are published or not. The documents may come from teaching and research institutions in France or abroad, or from public or private research centers.
L'archive ouverte pluridisciplinaire HAL, est destinée au dépôt et à la diffusion de documents scientifiques de niveau recherche, publiés ou non, émanant des établissements d'enseignement et de recherche français ou étrangers, des laboratoires publics ou privés. 


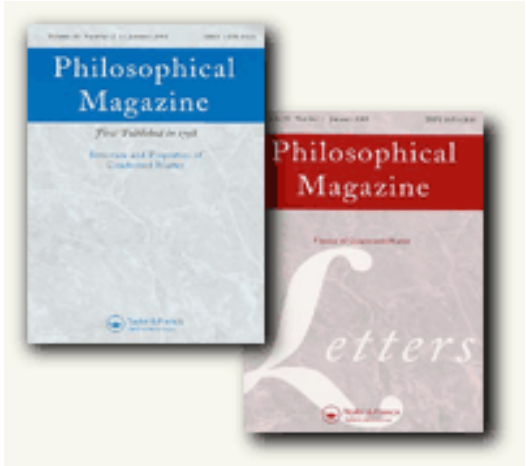

Transport mechanisms in 8-tris-hydroxyquinoline aluminium (Alq3) electronic layers: a study by photodipolar absorption

\begin{tabular}{|c|c|}
\hline Journal: & Philosophical Magazine \& Philosophical Magazine Letters \\
\hline Manuscript ID: & TPHM-06-Feb-0030 \\
\hline Journal Selection: & Philosophical Magazine \\
\hline $\begin{array}{r}\text { Date Submitted by the } \\
\text { Author: }\end{array}$ & 02-Feb-2006 \\
\hline Complete List of Authors: & $\begin{array}{l}\text { MOLITON, André; Facuty of Scuiences and Technics, UMOP } \\
\text { LUCAS, Bruno; Fac Sciences et Techniques, UMOP } \\
\text { Berthon, Sebastien; Fac Sciences et Techniques, UMOP } \\
\text { Rammal, Wassim; Fac Sciences et Techniques, UMOP } \\
\text { HIORNS, Roger; LPCP, Chimie }\end{array}$ \\
\hline Keywords: & transport, aluminium, absorption \\
\hline Keywords (user supplied): & transport mechanisms, photodipolar absorption \\
\hline
\end{tabular}

\section{(s) ScholaroNE \\ Manuscript Central}




\title{
Transport mechanisms in 8-tris-hydroxyquinoline aluminium (Alq3) electronic layers: a study by photodipolar absorption
}

\author{
André MOLITON*, Bruno LUCAS, Sébastien BERTHON, Wassim RAMMAL, \\ UMOP, CNRS - FRE 2701, Faculté des Sciences et Techniques, \\ 87060 - Limoges cedex, France. \\ * e-mail : amoliton@unilim.fr \\ *Tel: +33 (0)555 457 432; Fax: +33 (0)555 457288 \\ and Roger C. HIORNS \\ Laboratoire de Physico-Chimie des Polymères, CNRS - UMR 5067, \\ Hélioparc Pau-Pyrénées, 2 Avenue du Président Angot, 64053 Pau cedex 9, France
}

\begin{abstract}
This paper describes the role of traps in the electronic conductivity of 8-tris-hydroxyquinoline aluminium in a conventional sandwich structure with indium tin oxide and aluminium electrodes. New results obtained by photodipolar absorption techniques and impedance spectroscopy are presented. The former method acts as a probe to highlight the role of traps. It is shown that optical pumping of electrons to trap levels gives a clear increase in dielectric absorption due to the reorientation of dipoles associated with trapped charges. The trap depth is estimated to be around $E_{\mathrm{t}}=0.19 \mathrm{eV}$, a value in good agreement with theoretical calculations and thermoluminescence measurements. The latter method permits a representation of the sample in terms of a circuit composed of a parallel capacitor $\left(C_{\mathrm{p}}\right)$ and resistor $\left(R_{\mathrm{p}}\right)$ both in series with a resistor $R_{\mathrm{S}} \approx 50 \Omega$ located on the anode side. A logarithmic plot of $R_{\mathrm{p}}$ as a function of the dc bias voltage gives a linear law that is recognized, for the first time, to be a consequence of a trapped charge limited (TCL) current. The linearity can be improved by the introduction of a field-dependent mobility.
\end{abstract}




\section{Introduction}

In the development of molecular materials for organic optoelectronics, particularly for applications using electroluminescence, the electron emitter 8-tris-hydroxyquinoline aluminium (Alq3) has been studied in depth [1]. Figure 1 shows the chemical structure of this material which is an organometallic complex based on a central metal cation coordinated to quinolate ligands. It is generally prepared as a thin film by evaporation under vacuum, which in itself demands that the complexes exhibit no overall charge or saturated coordination numbers [2]. Alq3 satisfies these requirements and is the most widely used compound, even if its fluorescent quantum yield is relatively low. It provides films with a stable electroluminescence and is a good transporter of electrons.

[Insert Figure 1 about here please]

Numerous discussions have taken place concerning the electronic conduction mechanisms and the role of traps in Alq3 [3]. For example, a trapped charge limited (TCL) model in which the trapping time is longer than the average transit time for a hop between the LUMOs of two molecules, was proposed to answer criticisms concerning electronic transport in a band structure where there is a sufficient delocalization of carriers to establish a thermal equilibrium between free and trapped charges [4].

In this paper, we present new results, which, on interpretation, advocate the use of conductivity laws involving trap mechanisms. Experimental results are based on photodipolar absorption measurements obtained from thermo-photo-dielectric effects, as currently used with inorganic materials (class II-VI semiconductors such as $\mathrm{ZnS}(\mathrm{Cu})$ ). This technique has not been used before with Alq3 to our knowledge, and is here complemented by the use of impedance spectroscopy. We discuss the possibility of a modified TCL law to take into account a dependence of the mobility on applied electrical field.

\section{Electronic structure and transport mechanisms in an indium tin oxide/Alq3/Ca(Al) sandwich device}

\subsection{Energy levels in Alq3}

In polymers each monomer is joined to its neighbours by strong bonds, which have associated with them transfer integrals $\beta$ [5]. However, in thin films composed of molecules, cohesion arises from weak Van der Waals forces between weakly overlapping orbitals of the molecules. In addition, the greater the distances between the molecules, the weaker the 
[Insert Figure 2 about here please]

Figure 3 shows absorption and electroluminescence spectra of Alq3. The electroluminescence peak is red shifted by $\approx 0.4 \mathrm{eV}$ from the absorption peak. This is a Franck-Coulomb shift results from large conformational changes on optical excitation. These conformational changes, arising from strong electron-phonon coupling, broaden the electroluminescence spectrum and give rise to discrete trapping levels in the HOMO-LUMO gap.

[Insert Figure 3 about here please]

\subsection{Interface barriers}

The work functions of indium tin oxide (ITO), calcium and Alq3 are denoted by $W_{\text {ITO }}, W_{\mathrm{Ca}}$ and $W_{\mathrm{Alq} 3}$, and the electron affinity, the ionization energy and the size of the forbidden band of Alq3 by $\chi_{\mathrm{Alq} 3}, I_{\mathrm{PAlq} 3}$ and $E_{\mathrm{GAlq} 3}$, respectively. Experimental values are $W_{\mathrm{ITO}}=4.6 \mathrm{eV}, W_{\mathrm{Ca}}$ 
$=2.9 \mathrm{eV}, \chi_{\mathrm{Alq} 3}=3.3 \mathrm{eV}, E_{\mathrm{GAlq} 3}=2.6 \mathrm{eV}$, so that $I_{\mathrm{PAlq} 3}=\chi_{\mathrm{Alq} 3}+E_{\mathrm{GAlq} 3}=5.9 \mathrm{eV}$ (values for Alq3 were obtained from Schmidt et al. [8]).

Organic light-emitting diodes (OLED)s are generally fabricated as detailed in Figure 4 (a) [9]. Once contact is made with the electrodes, the bands move through the adjustment of Fermi levels such that $V_{\mathrm{FB}}=\left(W_{\text {IтO }}-W_{\mathrm{Ca}}\right) / q$ where $V_{\mathrm{FB}}$ is the voltage difference of the flatbands and $V_{\text {app }}$ is the applied dc bias voltage (Figure 4(b)). The drop in voltage that appears of its own accord is in fact what we term $V_{\mathrm{PS}}$ (the 'positive supplementary' voltage applied to the ITO anode), and is equal to the total applied voltage minus the flat-band voltage $\left(V_{\mathrm{FB}}\right)$. In this example $V_{\mathrm{FB}} \approx 2 \mathrm{~V}$, a non-negligible value with respect to those generally used (of the order of 0 to $15 \mathrm{~V}$ ).

Important information can be gained about the interfaces. The barrier at the anode $\left(\Delta_{\mathrm{A}}\right)$, observed by holes, is equal to $\Delta_{\mathrm{A}}=I_{\text {PAlq3 }}-W_{\text {ITO }}$. In this example $\Delta_{\mathrm{A}}=1.3 \mathrm{eV}$. It is more than probable that the contact at the anode is not ohmic and risks being limited by emissions from the anode. At the cathode there is no barrier to the injection of electrons. The drop in potential energy for electrons $\Delta_{\mathrm{C}}=\chi_{\mathrm{Alq} 3}-W_{\mathrm{Ca}}$, which gives $\Delta_{\mathrm{C}}=0.4 \mathrm{eV}$. This contact can be assumed to be ohmic because near the electrode the Fermi level penetrates the Alq3 LUMO.

[Insert Figure 4 about here please]

\subsection{Transport mechanisms}

\subsubsection{Introduction}

Various mechanisms can be envisaged for charge transport. The voltage and current flow characteristics of a material are usually studied and explained in terms of charge injection under an electric field (Schottky effect) or by tunnelling, in which case the current is electrode-limited. However, as here, the results may be interpreted using models based on current flow through the bulk of the material. It is important to note though that in order to reduce the threshold potential of the device $\left(\mathrm{ITO} / \mathrm{Alq}_{3} / \mathrm{Ca}(\mathrm{Al})\right)$, the effect of the metal used for the electrode or the mobility of carriers within the organic layer should be characterized depending on the technique used.

Studies of $I(V)$ characteristics have shown that Schottky emissions can give an ideality factor $n$ of the order of 20 (a very high value indeed) and an unusually high diode series resistance of $50 \mathrm{k} \Omega$. However, the emission law for tunnelling effects (Fowler-Nordheim law) is not completely verified $[4,10]$. In fact, it is the Alq3 bulk that limits the current flow 
and furthermore, the conductivity cannot arise from carriers in extended states. In fact, the $I(V)$ characteristic follows the TCL relation $I \propto V^{m+1} / d^{2 m+1}$, notably within domains that yield electroluminescence.

\subsubsection{Conduction processes and trapping levels}

Qualitatively, in $\mathrm{Alq} 3$, the $\mathrm{Al}^{3+}$ ion complexes with the 3 ligands to leave them with the capability, through back bonding, to act as effective electron acceptors. This character results in the easy propagation of electrons between molecules. The material is therefore considered to be n-type.

The existence of extended states in condensed small molecules is controversial. As mentioned above, semi-empirical calculations have been used to determine the energy levels for an isolated molecule. However, the weak Van der Waals intermolecular interactions do not even yield narrow molecular bands. When dealing with electroluminescence, the continuity (or non-continuity) of energy levels susceptible to receiving injected charge carriers at interfaces is problematic. If the energy separation between levels is too great, then there is a risk that any injected charge will not find an accepting energy level. Given the size of the molecule however, it is probable that there is a large number of energy levels and consequently these should be sufficiently close to ensure that charge carriers are not blocked. Even if there are permitted bands arising from weak Van der Waals bonding, the very limited overlapping of molecular orbitals makes these bands narrows $(B<k T)$ and the effective-mass approximation can no longer be applied to evaluate charge mobility.

By assuming that any collision is isotropic, and therefore that the relaxation time is equivalent to the time between two consecutive collisions, a general equation for the mobility $\mu$ [11] leads-with the Ioffe and Regel rule $l>a$ (where $a$ represents an average intermolecular distance and $l$ the mean free pathway of an electron) [12] - to the inequality $\mu$ $>q a^{2} / \hbar$ [9]. Given that $a \approx 5 \times 10^{-8} \mathrm{~cm}$, we find that $\mu \geq 1$ to $10 \mathrm{~cm}^{2} \mathrm{~V}^{-1} \mathrm{~s}^{-1}$. With $\mu \ll 1 \mathrm{~cm}^{2}$ $\mathrm{V}^{-1} \mathrm{~s}^{-1}\left(\mu \approx 5 \times 10^{-5} \mathrm{~cm}^{2} \mathrm{~V}^{-1} \mathrm{~s}^{-1}\right.$ in Alq3), we do not have the inequality required (i.e. $\mu \geq 1$ to $\left.10 \mathrm{~cm}^{2} \mathrm{~V}^{-1} \mathrm{~s}^{-1}\right)$ to obtain conduction through delocalized states.

Structural defects resulting from electron injection into Alq3 have been studied. According to Section 2.1, an injected electron should enter the first empty level localized around pyridine groups. In order to determine the approximate trapping energy associated with an electron injected into Alq3, calculations were performed for a neutral Alq3 with the geometric structure of its anion [13]. Transition energies of 3.07 to $3.20 \mathrm{eV}$ and $3.34 \mathrm{eV}$ underwent a large red shift of the order of $0.21 \mathrm{eV}$ with respect to the neutral and fundamental 
state of Alq3, a value which represents the electron trap energy depth. It was also thought that these calculations provided insight into the nature of the traps in Alq3. When an additional electron is localized on a molecule it occupies anti-bonding orbitals and the molecule accordingly relaxes into a new structure (Frank-Condon principle). This relaxation towards a lower energy level can be used to automatically trap an electron that would otherwise move to a neighbouring molecule through polaronic processes. Given that there are many possible variants to the Alq3 structure, an exponential breakdown of trap levels leading to distribution of levels in the LUMO band is possible. The upshot of this is that conduction mechanisms associated with trap levels can therefore appear in otherwise resistive media.

In the next section, we briefly recall the main results for current flow obtained with electrical conduction involving traps. For the discussion section, a clear understanding of the mechanisms leading to the physical laws is of importance.

\subsubsection{Transport mechanisms in resistive organic materials with traps; effective mobility}

\subsection{3a The space-charge-limited-current law without traps}

The current can be space-charge limited when the contact is ohmic. Given that the Alq3 is close to being an insulator, space-charge formation near the electrode interface opposes the trickle of current through the material. At a sufficiently high applied field $\left(E_{\mathrm{a}}\right)$, the space charge is pressed right back to the interface and can effectively enforce a saturation current. At this point, it is important to recall the hypotheses leading to the common space-chargelimited (SCL) law. First, the band model is applicable to the treatment of injection of carriers with a current not limited by the electrode. While considerable controversy does surround the question of whether or not band models can be applied to organic solids, a model does indeed exist for these [5,14], which is close to that proposed for amorphous semiconductors [15]. The most important assumption of the model is that the energy levels within an insulator, localized or not, can accept injected charges at the interface. Second, carrier mobilities are assumed to be independent of $E_{\mathrm{a}}$ and the dielectric permittivity $(\varepsilon)$ is not modified by charge injection. Poole-Frenkel or impact ionization effects are not considered. Third, the electric field is assumed to be high enough to render negligible the following current components:

- ohmic current due to thermally generated carriers with density $n_{0}$ as under a high injection regime $n>n_{0}$;

- current due to diffusion, because the applied potential is considerably greater than the thermal potential $k T / q$. 


\subsection{3b The effect of traps: the effective mobility and the modified SCL law}

If $n$ is the density of free charges with mobility $\mu_{\mathrm{n}}$, and $n_{\mathrm{t}}$ the density of trapped charges on a discrete level $E_{\mathrm{t}}$, then the current density $(J)$ can be written either as $J=q n \mu_{\mathrm{n}} E_{\mathrm{a}}$ or as $J=q$ $\left(n+n_{\mathrm{t}}\right) \mu_{\text {eff }} E_{\mathrm{a}}$ which defines the effective mobility $\left(\mu_{\mathrm{eff}}\right)$. The current density with traps $\left(J_{\mathrm{sT}}\right)$ becomes

$$
J_{\mathrm{sT}}=\frac{9}{8} \varepsilon \mu_{\mathrm{eff}} \frac{V_{\mathrm{a}}^{2}}{d^{3}}=\frac{9}{8} \varepsilon \mu_{\mathrm{eff}} \frac{E_{\mathrm{a}}^{2}}{d}
$$

Traps can be distributed according to a Gaussian law. Accordingly, the density of traps per unit energy $(g(E))$, centred about energy $E$, is of the form

$$
g(E)=\left(N_{\mathrm{t}} / k T_{\mathrm{t}}\right) \exp \left(-\left[E_{\mathrm{c}}-E\right] / k T_{\mathrm{t}}\right)
$$

in which $N_{\mathrm{t}}$ is the total density of trap levels and $T_{\mathrm{t}}$ is a constant characteristic of the distribution. Taking $T_{\mathrm{t}}=E_{\mathrm{t}} / k=m T$ in which $E_{\mathrm{t}}$ represents the characteristic energy of traps with respect to $E_{\mathrm{C}}$ and $m$ a characteristic of the trap distribution, the current density is generally TCL and given by

$$
J \equiv J_{\mathrm{TCL}} \propto V^{m+1} / d^{2 m+1}
$$

Qualitatively, an ohmic law is followed at low voltages (due to thermally generated carriers of density $n$ ) in thick films. As the voltage increases, the Fermi pseudo-level rises and traps start to fill. This decrease in the number of empty traps, which can now receive few electrons, induces a rapid increase in the mobility and the overall current, resulting in the TCL law being observed $\left(I \propto V^{m+1} / d^{2 m+1}\right)$. At a sufficiently high value of $V$, all traps are filled and the classic SCL law may be observed. However, such values for $V$ are rarely obtained without destroying the films.

Numerous arguments have been developed as to why the TCL law is observed [4] but controversial points have been raised [3], as will now be discussed.

\subsubsection{Controversial points}


Apart from doubts concerning the validity of the band scheme for small molecules, another controversial point relates to the hypothesis of the invariance of carrier mobility with respect to an electric field, which is used in establishing the SCl and TCL laws. Because of the low mobilities and short mean free paths in organic solids, Kao and Hwang proposed that, between successive collisions, the carriers could not gain much energy from an applied field, and hence their mobilities should be essentially independent of the applied field [16]. However, in disordered systems, general expressions for $\mu$ are obtained empirically and have only been verified through Monte Carlo simulations [17], or in accordance with an empirical law for charge transfer [18]. Carriers in small molecules generally exhibit a mobility which follows the Poole-Frenkel law in the form $\mu \propto \mu_{0} \exp \left(\alpha E_{\mathrm{a}}{ }^{1 / 2}\right)$. However, in certain materials, such as polymers doped with molecules to the order of 5 to $25 \%$ by mass, mobilities do decrease above a certain value of $E_{\mathrm{a}}$. As shown in Figure 5, in qualitative terms, we can suppose that this effect is due to 'diagonal disorder' associated with the variable distances between molecules [19]. Under a weak field, there are many available and facile pathways (short distance hops in different directions with respect to the field) which are, nevertheless, removed once a strong field is applied.

[Insert Figure 5 about here please]

\subsubsection{Alternative models}

By modelling the characteristics of organic materials such as Alq3, Ioannidis et al. showed that trapping levels need not be invoked [3]. This group even supposed that such levels do not exist, because in examples where only electrons have been injected (using the same contacts on either side, which are assumed to be ohmic), the $I(V)$ curves are identical whether or not the applied voltage is interrupted during the measurements, indicating negligible electron trapping. They consider that experimental characteristics may be based on Ohm's law, into which can be included an appropriate law for a variation in mobility of the form $\mu=$ $\mu_{0} \exp \left(\alpha E_{\mathrm{a}}{ }^{1 / 2}\right)$ where $\mu_{0}$ is the mobility under a weak field and $\alpha$ a parameter increasing with disorder. In their fitting, they found $\alpha=1.3 \times 10^{-2} \mathrm{~cm}^{0.5} \mathrm{~V}^{-0.5}$ and $\mu_{0}=6.5 \times 10^{-10} \mathrm{~cm}^{2} \mathrm{~V}^{-1} \mathrm{~s}^{-1}$.

It is important to state, however, that the presence of trapping levels has been confirmed using a variety of techniques. These include thermo-stimulated luminescence (TSL) spectroscopy which was demonstrated by Forsythe et al.[1] in the modelling of a principal peak using a distribution of traps with energies between 0.25 and $0.15 \mathrm{eV}$ (two 


\subsection{Experimental procedures}

Alq3, obtained from Aldrich, France, was deposited by thermal evaporation onto an ITO ( $c a$ $300 \mathrm{~nm}$ thick on a glass substrate and obtained from Merck, France) plate in a secondary vacuum ( $c a 10^{-6} \mathrm{mbar}$ ), and then covered with aluminium ( $c$ a $100 \mathrm{~nm}$ thick layer) by thermal deposition. Once the sample cell was prepared, with electrical contacts placed on opposite sides of the sample in order to prevent short-circuits as shown in Figure 6, it was transferred to the apparatus schematized in Figure 7. The experimental measurements were performed using a Hewlett-Packard 4274A bridge. Samples were cooled to $77 \mathrm{~K}$ and then data were obtained in the $20 \mathrm{~Hz}-1 \mathrm{MHz}$ frequency range during heating passages from $150 \mathrm{~K}$ to 320 K. A Wood lamp, Model B 100 AP UVP manufactured by Upland, USA, and supplied by Fisher Bioblock Scientific, France was used to supply the UV illumination at an intensity of $7000 \mu \mathrm{W} \mathrm{cm}{ }^{-2}$ and a wavelength $(\lambda)$ of $365 \mathrm{~nm}$ (figures supplied by the manufacturer in accordance with the filter used on the lamp). The sample was also exposed to ambient natural light.

[Insert Figures 6 and 7 about here please]

\subsection{Components observed by dielectric absorption spectroscopy}


Prior to considering the results from the dielectric experiments, it is worth discussing what has actually been measured. Since the dielectric absorption analyses cannot discriminate between the various electrical currents in the sample, in order to determine the true dielectric response of Alq3 the other components that are concurrently measured must also be determined [22]. In other words, effects due to the true dielectric loss, characterized by the imaginary component of the dielectric susceptibility $\left(\chi^{\prime \prime}\right)$, must be separated from those due to: the dc conduction $\left(\sigma_{0}\right)$ caused by free charges that are practically independent of frequency (at least in the low-frequency range); hopping conduction $\left(\sigma_{\mathrm{h}}\right)$; photoconduction $\left(\sigma_{\mathrm{ph}}\right)$ for an illuminated sample; and variations at the interfaces between Alq3 and the electrodes. For the measured apparent dielectric loss, or rather the imaginary component of the effective dielectric response $\left(\varepsilon^{\prime \prime}\right)$ it is therefore possible to write

$$
\varepsilon^{\prime \prime}=\chi^{\prime \prime}+\frac{\sigma}{\varepsilon_{0} \omega}
$$

where $\sigma=\sigma_{0}+\sigma_{h}+\sigma_{p h}$. Following from the discussion above, the last component $\left(\sigma / \varepsilon_{0} \omega\right)$ arises only because the instrument cannot distinguish between true dielectric and electronic transport processes. In the Debye model, the dielectric loss due to $\chi "$ can be represented as a double potential well as schematized in Figure 8 . Under the influence of an applied alternative electric field, the electrons oscillate around an equilibrium position between positions $\mathrm{A}$ and $\mathrm{B}$, which are separated by a potential barrier of height $U$. In the most simple scenario, the mechanism for the oscillation is a relaxation process of time constant $\tau$ where $\tau=\tau_{0} \exp (U / k T)$. When the dielectric loss is at a maximum, $\omega_{c} \tau=1$, and the activation energy $\mathrm{U}$ can be related to the trap depth by $U=E_{\mathrm{t}}$, as has been possible for luminescent inorganic II-VI semiconductors. It is possible to distinguish the component $\sigma_{0}$ from dielectric phenomena because $\varepsilon^{\prime \prime}(\omega)$ is proportional to $1 / \omega$ and, on decreasing $\omega$ towards $0 \mathrm{~Hz}$ when $\varepsilon^{\prime}(\omega)$ attains a constant value, the remaining measurement is due to $\sigma_{0}$ which becomes the dominant process. The component $\sigma_{\mathrm{h}}$ has often been observed in amorphous materials and various theories have been proposed to define it. The most widely used is $\sigma_{\mathrm{h}}=C \omega^{\mathrm{s}}$, where $s=$ $s(T)$ in accurate theories [15]. However, for amorphous materials with traps, the photoconductivity may be discerned as a function of temperature through the equation

$$
\sigma_{p h}=q \mu \tau_{T} G\left(N_{c} / N_{t}\right) \exp \left(-\left[E_{c}-E_{t}\right] / k T\right)
$$




\subsection{Results from dielectric experiments}

During illumination, excited electrons are 'pumped' from the HOMO to the trap levels, with a transient photo-electronic process around the LUMO levels as schematized in Figure 8. The results from the dielectric analyses of the sample are presented in Figure 9 (curves 1-4). Without illumination and under relatively fast heating, the sample gave a small and near constant response (curve 1). Again without illumination, but with slow heating, the dielectric response was weak with a broad peak at around $220 \mathrm{~K}$ caused by trapped electrons excited by ambient natural light (curve 2). Under illumination during both the cooling and heating passages, a component due to $\sigma_{\mathrm{h}}$, which eclipsed the dielectric contribution could be observed (curve 3). With illumination during only the initial cooling passage, the dielectric response could be clearly discerned at around $220 \mathrm{~K}$ on the heating passage (curve 4). It should be noted that as fluorescence is a near-instantaneous process with a relaxation time of less than 1 $\mathrm{ms}$, it would not have interfered with the data in curve 4 on account of the delay of several minutes between the illumination during the cooling passage and the measurement during the heating passage.

[Insert Figure 9 about here please]

Figure 10 shows curves for $\varepsilon^{\prime \prime}=f(T)$ at a range of frequencies for the samples exposed to the lamp light or only natural light during the cooling curve, respectively denoted (a) and (b). The dielectric peaks are at higher temperatures as the frequency increases, a characteristic of Debye dielectric absorption. The curve of $\log v_{\mathrm{c}}=\mathrm{f}(1 / T)$ shown in Figure 11(a) and derived from the (a) plots in Figure 10 permits a calculation of the activation energy 
$E_{\mathrm{t}} \approx 0.19 \mathrm{eV}$. This value is in good agreement with that obtained elsewhere by thermoluminescence studies $\left(0.15 \mathrm{eV}<E_{\mathrm{t}}<0.25 \mathrm{eV}\right)[1]$, TCL measurements $\left(E_{\mathrm{t}}=0.15 \mathrm{eV}\right)$ or theoretical calculations $\left(E_{\mathrm{t}}=0.21 \mathrm{eV}\right)$ [4]. From Figure 11(b), derived from the (b) plots of Figure 10, it was possible to conclude that electrons are trapped for a considerable time (several minutes) at the low temperatures used in this study; the level of the traps $\left(E_{\mathrm{t}}\right)$ was calculated to be $0.18 \mathrm{eV}$, and it is probable that they are nearly identical in nature to those filled by the action of the lamp light. It should be noted that to obtain reproducible curves, slow heating passages $\left(\mathrm{ca} 0.5^{\circ} \mathrm{C} \mathrm{min}^{-1}\right)$ were required.

[Insert Figures 10 and 11 about here please]

It is interesting to note that in going from $100 \mathrm{~Hz}$ to $1 \mathrm{kHz}$ there is a steady increase in the intensity of the Debye peaks shown in Figure 10. This increase is most probably due to a superposition of two effects. Firstly, the thermally activated process of photoconductivity, as detailed in Section 3.2, which gives rise to a large increase in conductivity at the higher temperatures indicated in Figure 9 (curve 3). Secondly, even if the excitation caused by UV is suppressed, as indicated in curve 3 and to a lesser extent in curve 2 where excitation is caused only by natural light, the photodipolar measurements can be influenced by a slow process of phosphorescence, which may be of non-negligible intensity for a period of several tens of minutes. The combination of the weak photoconductivity (without peaks) and the Debye absorption (that generates peaks) results in this behaviour; so, as the actual positions for the peaks remains unchanged in relation to a classical Debye behaviour, the calculated activation energies are nevertheless directly related to the photodipolar absorptions and therefore also the trap depths.

\subsection{Study of the contact resistance by impedance spectroscopy}

In order to calculate the contact resistance in the ITO/Alq3/Al device, impedance spectroscopy was used. Figure 12 shows a Cole-Cole plot at several dc bias voltages. The minimum value for $\operatorname{Re}(\mathrm{Z})$, obtained at the highest frequency, indicates that $R_{\mathrm{s}}$ has a value of ca $50 \Omega$. The maximum value for $\operatorname{Re}(\mathrm{Z})$, obtained at the lowest frequency, indicates the sum of $R_{\mathrm{s}}$ and $R_{\mathrm{p}}$, and therefore $R_{\mathrm{p}}$ can be calculated to be $c a 1.7 \times 10^{3} \Omega$ at zero dc bias. Given that experiments performed on similar devices made from ITO/Alq3/Au (in which the work functions of ITO and $\mathrm{Au}$ are of the same order) and Al/Alq3/Al indicated a value for $R_{\mathrm{S}}$ of 
around $50 \Omega$ and $15 \Omega$ respectively, it is possible to think that $R_{\mathrm{s}}$ is located at the anode side of the equivalent circuit. Importantly, the analysis made above also indicated that the interface capacitance was negligible, and the interfaces could therefore be assumed to have no air gaps or insulating barriers.

[Insert Figure 12 about here please]

\subsection{Discussion on possible improvements to the TCL law}

As traps have been shown to exist through photodipolar effects, it is useful to consider the accuracy of the TCL law and, indeed, how it may be improved. As shown in Figure 13, $R_{\mathrm{p}}$ decreases as the dc voltage increases while $C_{\mathrm{p}}\left(=1 / \omega_{\max } R_{\mathrm{p}}\right)$ is almost independent of the dc bias voltage. This observation was also made for a similar device based on PPV instead of Alq3 [24]. From the TCL law given by $J=K \frac{V^{m+1}}{d^{2 m+1}}$, where $K$ is a constant, it is possible to deduce the voltage dependence of the resistance $R_{\mathrm{p}}$ in such a way that $R_{p} \propto V / J \propto V^{-m}$. On plotting $\log R_{\mathrm{p}}$ against $\log V$, as in Figure 13 , it was found that $m \approx 2.5$. On making a first analysis, the reasonable linearity of the $R_{\mathrm{p}}$ versus $\log V$ plot would seem to indicate that the conductance followed the TCL law owing to an exponential trap distribution. However, on further inspection, it can be noticed that the plots in Figure 13 do not follow perfectly straight lines. Indeed, plots of $\log J$ against $\log V$ elsewhere in the literature are also not linear [10]. It should also be noted that the latter results did not exhibit the Fohler-Nordheim law, which would indicate that the conductivity was bulk limited. Therefore, there is every possibility that an improvement can be made to the TCL law. By taking into account the dependence of mobility on temperature and electric field, it seems reasonable that effects due to space charges and the applied electric field should affect $J(V)$ characteristics [25].

[Insert Figure 13 about here please]

On reconsidering the general expression for current density, and ignoring the term for diffusion, $J=\rho v=q n \mu E$, where $n$ is limited by space charge. The equation may be rewritten in the form $J=q n \mu V / d$, and $n$ is generally proportional to $V^{m}$, where $m=1$ when only free untrapped charges contribute to the space charge. This in effect gives rise to the saturation current $\left(J_{\mathrm{s}}\right)$ as indicated in the Mott-Gurney law. However, if the space charge is dominated 
by trapped charges, as here, then, with traps distributed according to an exponential energy curve, $m=T_{\mathrm{c}} / T$ for which $E_{\mathrm{t}}=k T_{\mathrm{c}}$. While $J \propto V^{m+1}$, the $J(V)$ law follows variations in $\mu$. As the latter is not a constant, then $J$ will no longer follow a law based on $V^{m+1}$.

The problem therefore is to resolve whether space charge or mobility is dominant in any specific voltage domain. If it can be supposed that mobility varies slowly enough with respect to $E_{\mathrm{a}}$, so that an integration of Laplace's equation may be performed (Section 2.3.3 $(\mathrm{a}, \mathrm{b}))$, the appropriate law for the mobility, i.e. $\mu\left(E_{\mathrm{a}}, T\right)$, may be introduced into the TCL equation. If, however, the mobility varies strongly with respect to $E_{\mathrm{a}}$, the integration of Laplace's equation becomes problematic. While attempts to obtain a field-dependent mobility from space-charge-limited-current variations against voltage have been presented elsewhere [26], the complexity of such equations make direct numerical treatment impossible, negating any contribution to the hypothesis of conduction by traps.

\section{Conclusions}

The presented experimental results and a consideration of the electronic structure of Aq3 have indicated that the conduction mechanism in a device based on ITO/Alq3/Al is bulk limited.

The TCL law would initially seem the most appropriate to describe electron transport in the device; however, improvements could be made to this law by bringing in a term to describe field-dependent mobility. Photodipolar absorption measurements indicated by a dielectric effect the presence of traps with depths of around $E_{\mathrm{t}}=0.2 \mathrm{eV}$. Optical pumping of electrons to trap levels gave a clear increase in the dielectric absorption. This method may therefore be used effectively to probe traps and demonstrates that the once controversial hypothesis that traps are present in Alq3 has been affirmed. 


\section{References}

[1] E.W. Forsythe, D.C. Morton, C.W. Tang Y. and Gao, paper presented at the SPIE conference, San Diego, USA, 3476123 (1998).

[2] S. Miyata and S. Nalwa, Organic electroluminescent materials and devices, (Gordon and Breach Science Publishers, Amsterdam, 1997).

[3] A. Ioannidis, E.W. Forsythe, Y. Gao, M.W. Wu, E.M. Conwell, Apl. Phys. Lett. 723038 (1998).

[4] P.E. Burrows, Z. Shen, V. Bulovic, D.M. McCarty, S.R. Forrest, J.A. Cronin and M.E. Thompson, J. Appl. Phys. 797991 (1996).

[5] A. Moliton, B. Lucas, C. Moreau, R.H. Friend and B. François, Phil. Mag. B 69(6) 1155 (1994).

[6] M. Pope and C.E. Swenberg, Electronic processes in organic crystals (Oxford, Clarendon Press 1982).

[7] J.L. Rivail, Eléments de chimie quantique, (Paris, InterEditions/Editions du CNRS, 1989).

[8] A. Schmidt, M.L. Anderson and N.R. Armstrong, J. Appl. Phys. 78(9) 5619 (1995).

[9] A. Moliton, Optolectronics of molecules and polymers, translated by R.C. Hiorns (Springer, New York, in press; from the original work, Optoélectronique moléculaire et polymère : des concepts aux composant (Paris, Springer France, 2003)).

[10] R. Antony, A. Moliton, B. Ratier and C. Moussant, Eur. Phys. J. AP 445 (1998).

[11] J.D. Wright, Molecular crystals, (Cambridge University Press, Cambridge, 1995).

[12] A.F. Ioffe and A.R. Regel Non crystalline, amorphous and liquid electronic semiconductors, in Progress in Semiconductors, vol.4, (Heywood and Co Ltd., London, 1960).

[13] S.R. Forrest, P.E. Burrows and M.E. Thomson in Organic electroluminescent materials and devices, edited by S. Miyata and H.S. Nalwa (Gordon and Breach Science Publishers, Amsterdam, 1997).

[14] P. Pflüger, G. Weiser, G. Campbell, J. Scott and B. Street, in Handbook of conducting polymers, vol. 2, edited by T.A. Skotheim, (Dekker, New York, 1986).

[15] N.F. Mott and E.A. Davis, Electronic processes in non-crystalline materials, (Clarendon Press, Oxford 1979).

[16] K.C. Kao, W. Hwang, Electrical transport in solids, (Pergamon Press, Oxford 1981).

[17] H. Bässler, Phys. Stat. Sol, B175, 15 (1993).

[18] W.D. Gill, J. Appl. Phys. 43(12), 5033 (1972). 
[19] N.C. Greenham and R.H. Friend, in Semiconductor Device Physics of Conjugated Polymers, edited by H. Ehrenheich, (Academic Press, 1995).

[20] M. Meier, S. Karg, K. Zuleeg, W. Brütting and M. Schwoerer, J. Appl. Phys. 84(1) 87 (1998).

[21] T.P. Nguyen, P. Molinie and P. Destruel, in Handbook of Advanced Electronic and Photonic Materials and Devices, edited by H.S. Nalwa (Academic Press, 2001).

[22] A.K. Jonscher, Dielectric relaxation in solids (Chelsea Dielectric Press, London, 1983).

[23] K. Morigaki, 194, Physics of amorphous semiconductors, (World Scientific, Imperial College Press, London, 1999).

[24] S.H. Kim, K.H. Choi, H.M. Lee, D.H. Hwang, L.M. Do, H.Y. Chu, T. Zyung, J. Appl. Phys. 87(2) 882 (2000).

[25] J. Shen, F. So, J.Yang, J.H. Xu, V.E. Choong and H.C. Lee, paper presented at the SPIE conference, San Diego, USA, 3476196 (1998).

[26] D. Natali and M. Sampietro J. Appl. Phys. 92(9), 5310 (2002). 


\section{Figure Captions}

Figure 1. Chemical structure of Alq3.

Figure 2. Localization of full (HOMO) and empty (LUMO) orbitals on Alq3.

Figure 3. Absorption and electroluminescence spectra of Alq3.

Figure 4. (a) Typical set up for an OLED based on ITO/Alq 3/Ca in which the ITO layer is about $300 \mathrm{~nm}$ thick, the Alq3 film is around $75 \mathrm{~nm}$, the calcium is approximately $250 \mathrm{~nm}$ and the aluminium electrode is $c a 75 \mathrm{~nm}$; and (b): the associated band scheme.

Figure 5. Qualitative description of charge transport under strong and weak fields. With a strong field, collisions are orientated and electrons are forced to follow a certain trajectory. On meeting a barrier, their passage is arrested, and thus mobility decreases when $E_{\mathrm{a}}$ is very high. With a weak field, however, trajectories are such that collisions are disorientated and electrons follow energetically favourable paths. In effect, transport is independent of positional disorder.

Figure 6. Schematization of the sandwich device. Note placement of electrodes to minimize possibility of short circuits.

Figure 7. Apparatus for photodipolar study.

Figure 8. Band scheme showing the position and depth of traps.

Figure 9. Curves plotted for $\varepsilon^{\prime \prime}=\mathrm{f}(T)$ at $v=40 \mathrm{~Hz}$ with the sample under various conditions: (1) no illumination and fast heating $\left(2 \mathrm{~K} \mathrm{~min}^{-1}\right)$; (2) no illumination and slow heating $\left(0.5 \mathrm{~K} \mathrm{~min}^{-1}\right)$; (3) illumination during cooling and heating (2 $\left.\mathrm{K} \mathrm{min}^{-1}\right)$; and (4) illumination during cooling passage only $\left(2 \mathrm{~K} \mathrm{~min}^{-1}\right)$.

Figure 10. Curves for $\varepsilon^{\prime \prime}=\mathrm{f}(T)$ at various frequencies for samples exposed to UV (curve a) or natural light only (curve b) during the cooling passages only.

Figure 11. Trap depths for samples exposed only during the cooling process to: (a) UV and natural light; and (b) natural light only.

Figure 12. Cole-Cole plot of the ITO/Alq3/Al device at several bias voltages.

Figure 13. Plots of $\log R_{\mathrm{p}}$ versus $\log V$. 


\section{Figure 1}

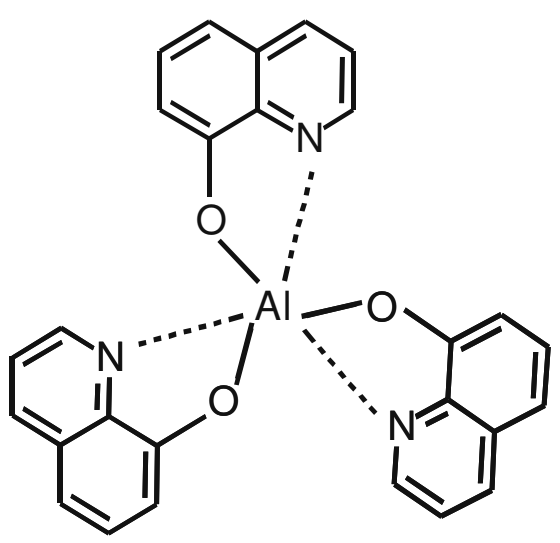




\section{Figure 2}

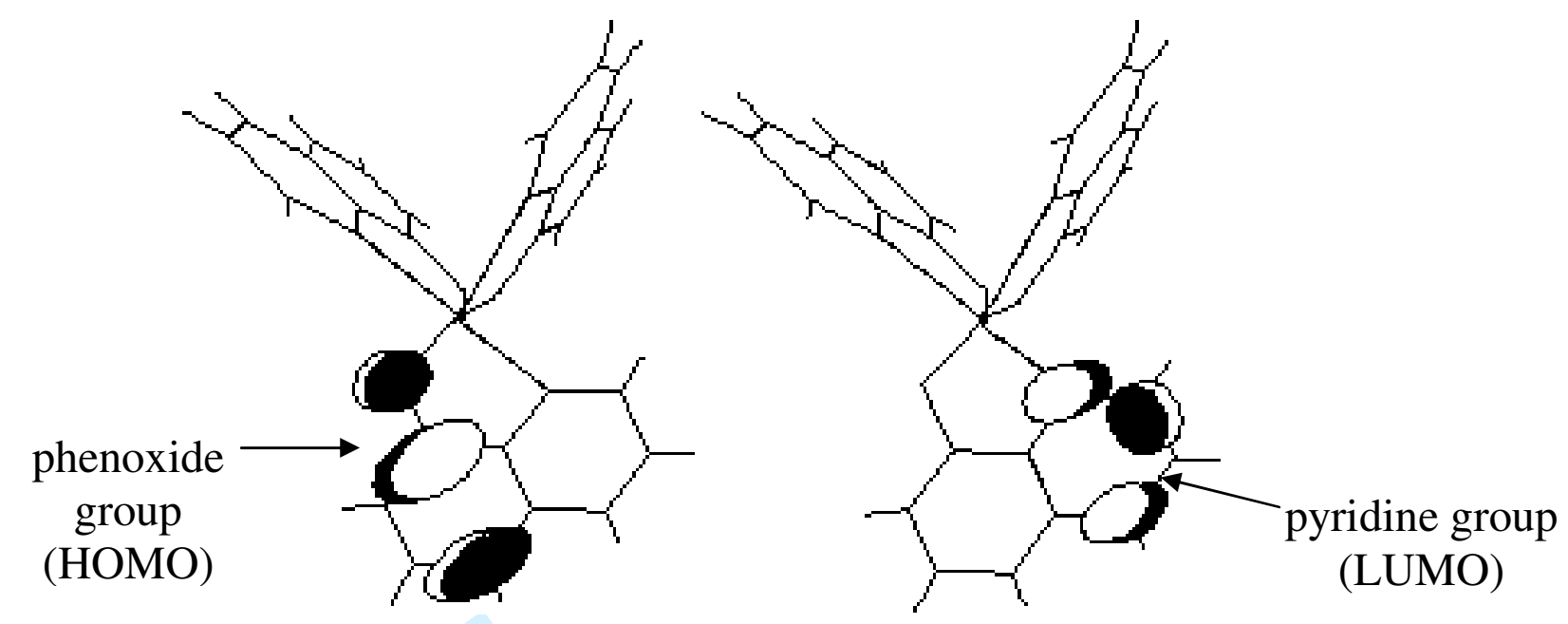


Figure 3

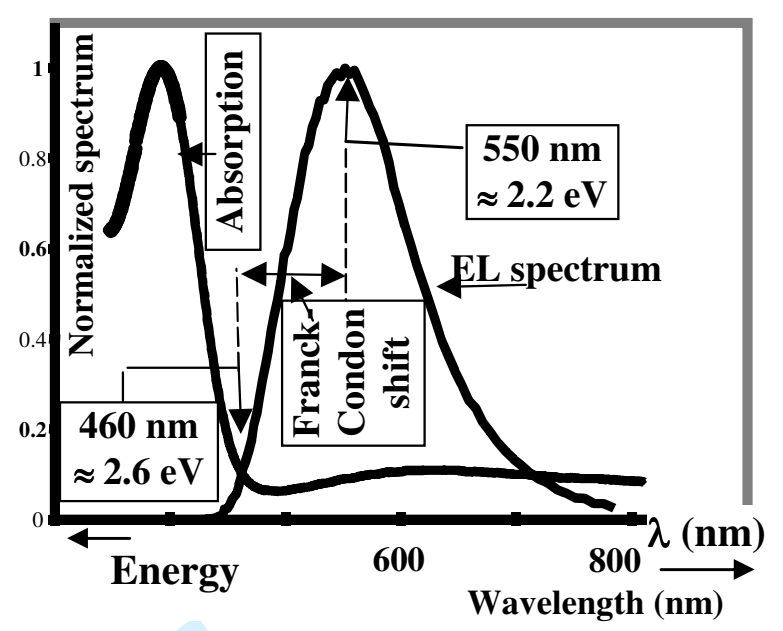


Figure 4

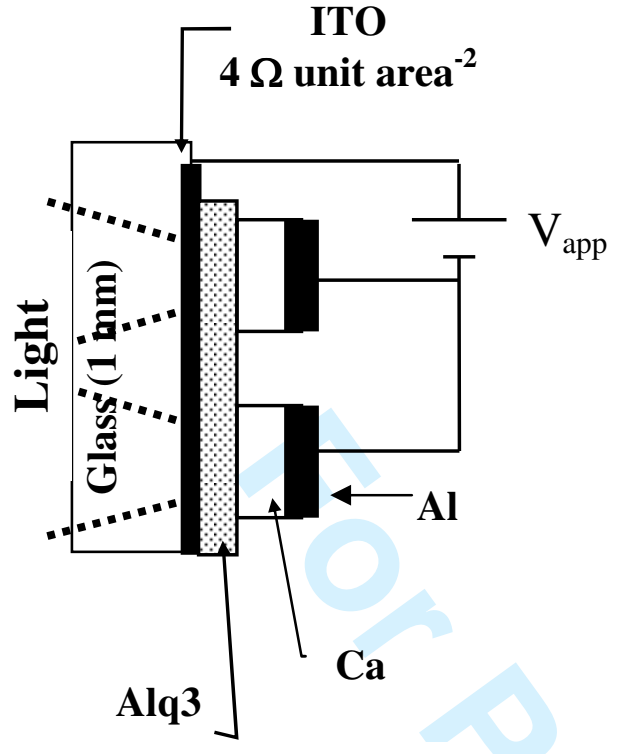

(a)

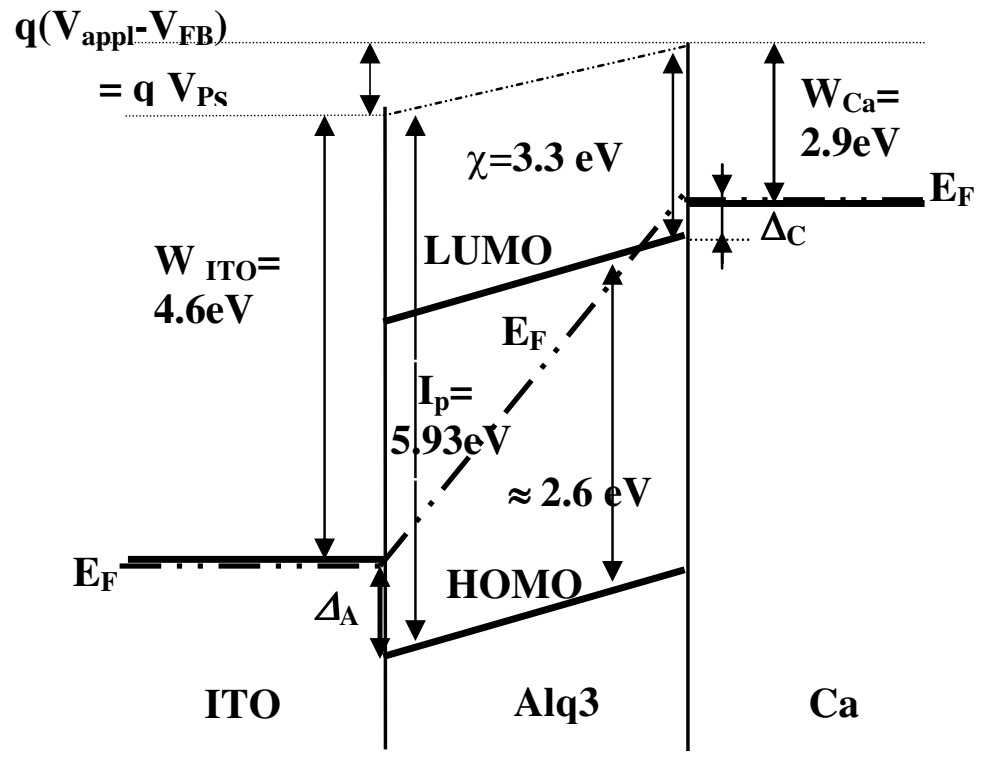

(b) 


\section{Figure 5}

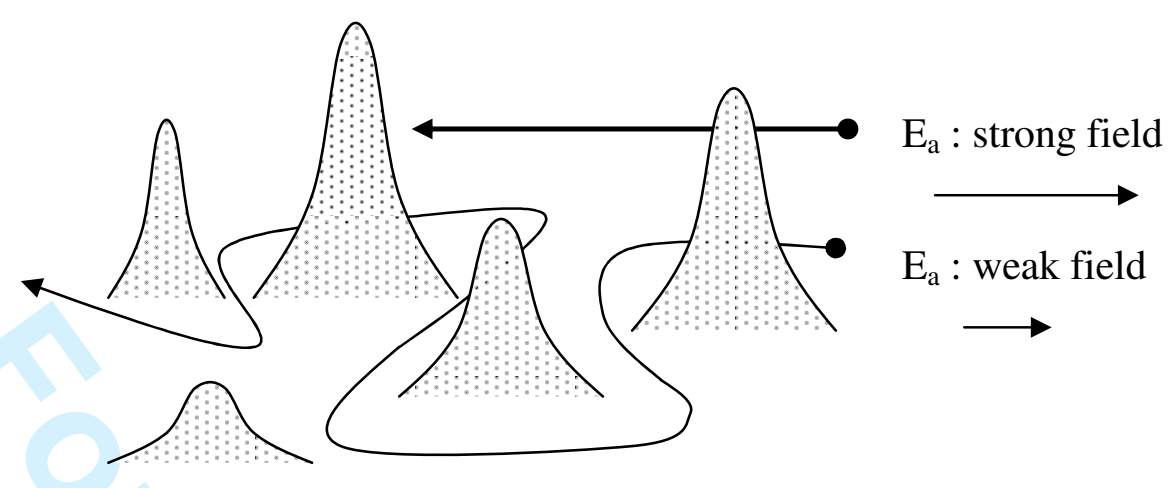




\section{Figure 6}

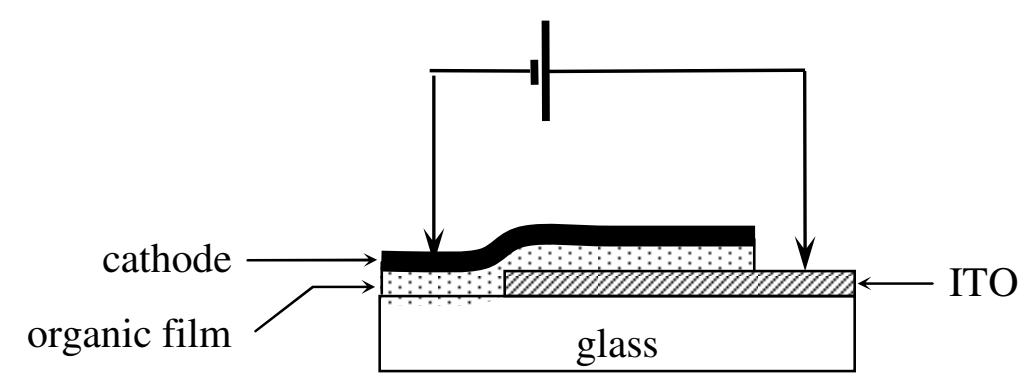


Figure 7

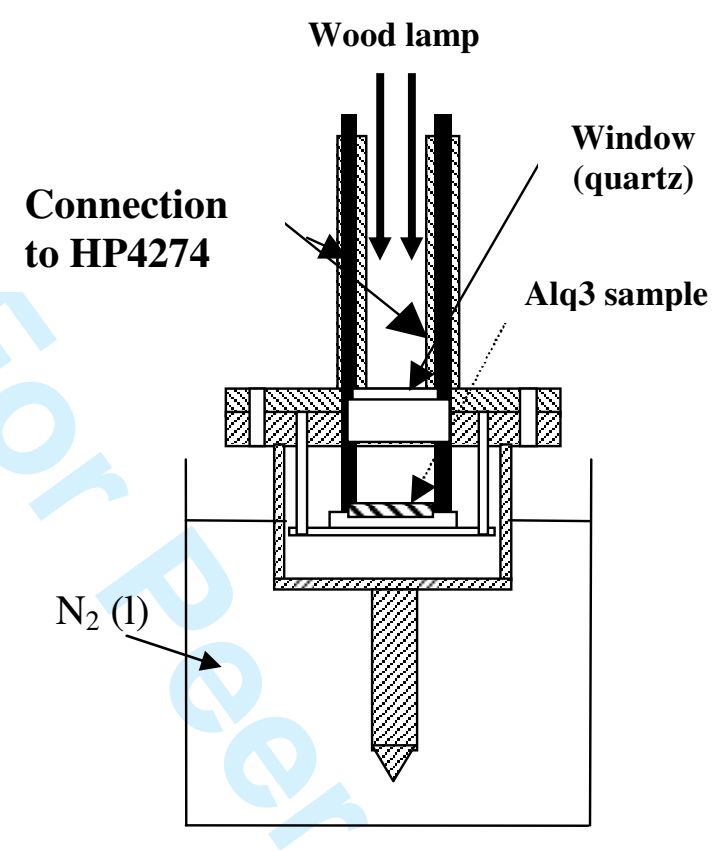




\section{Figure 8}

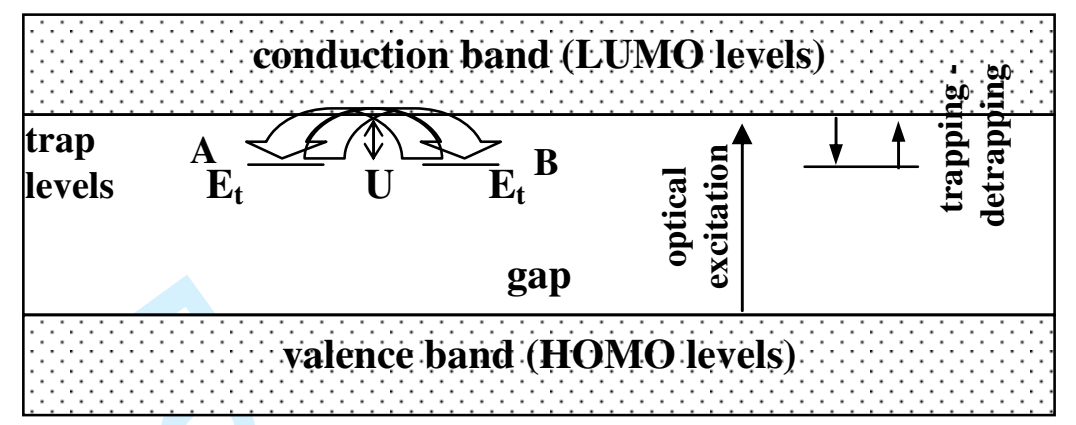


Figure 9

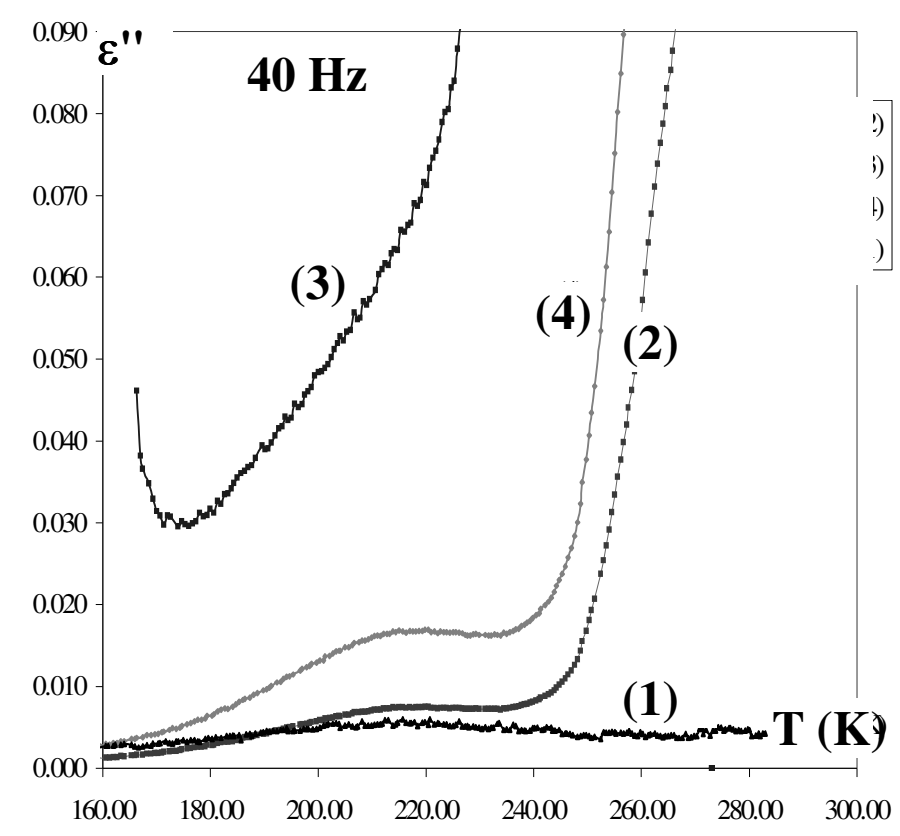


Figure 10
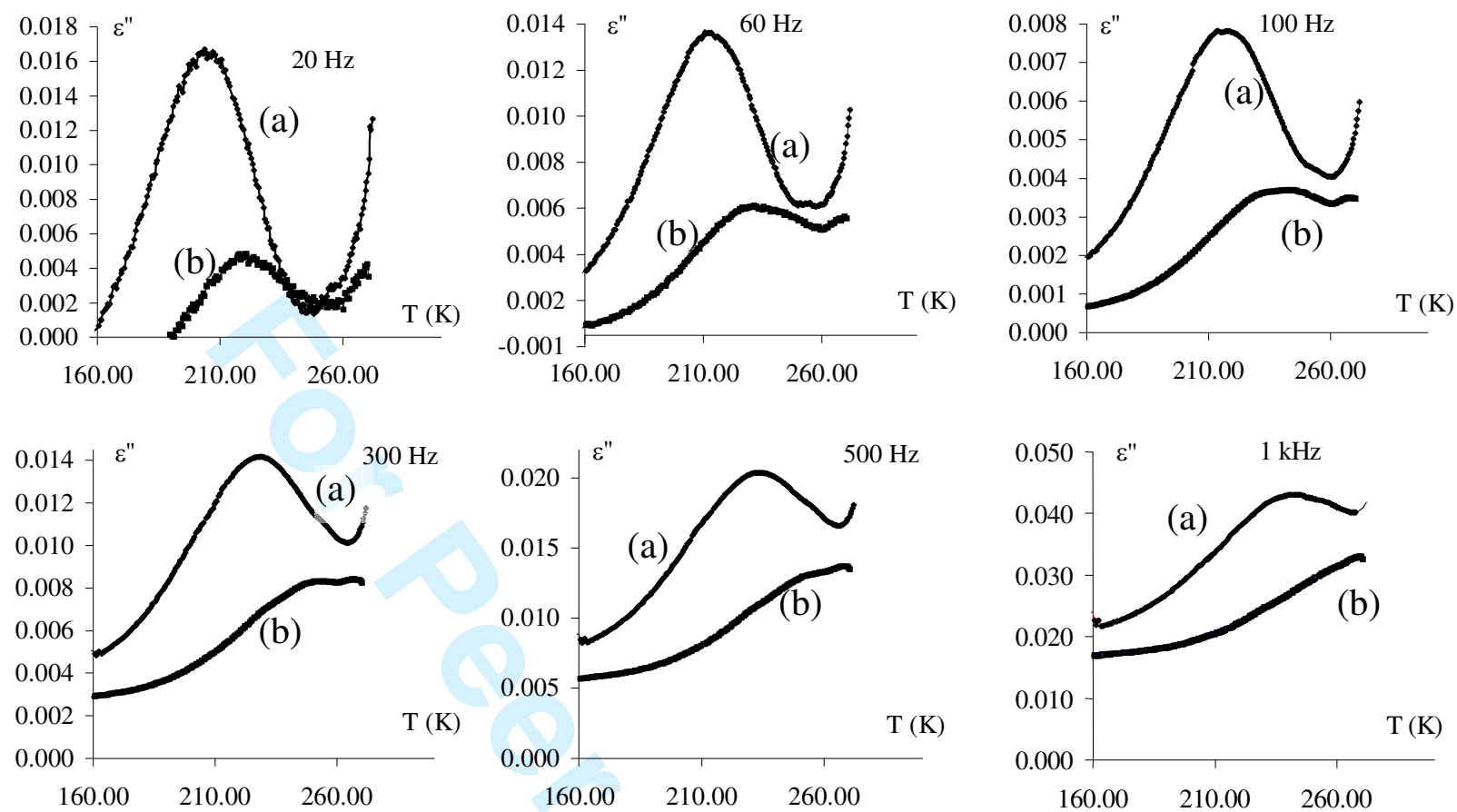


\section{Figure 11}

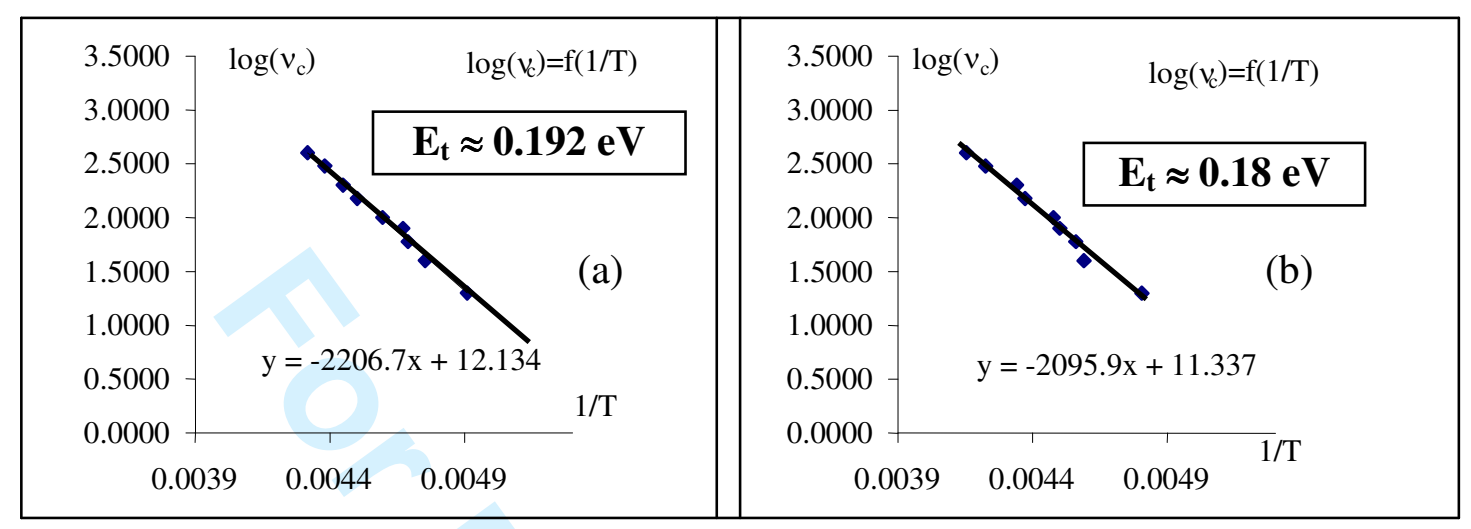




\section{Figure 12}

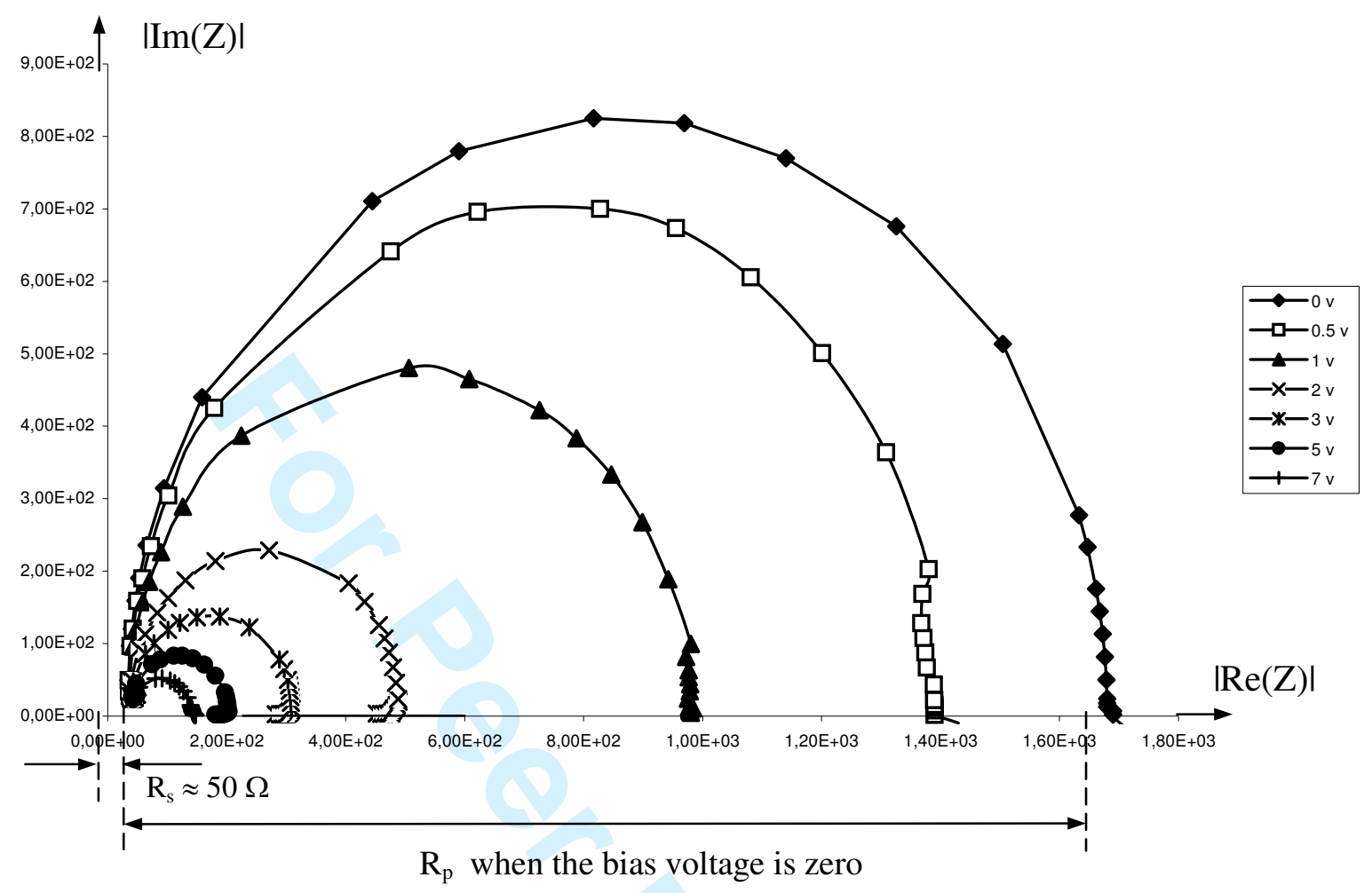




\section{Figure 13}

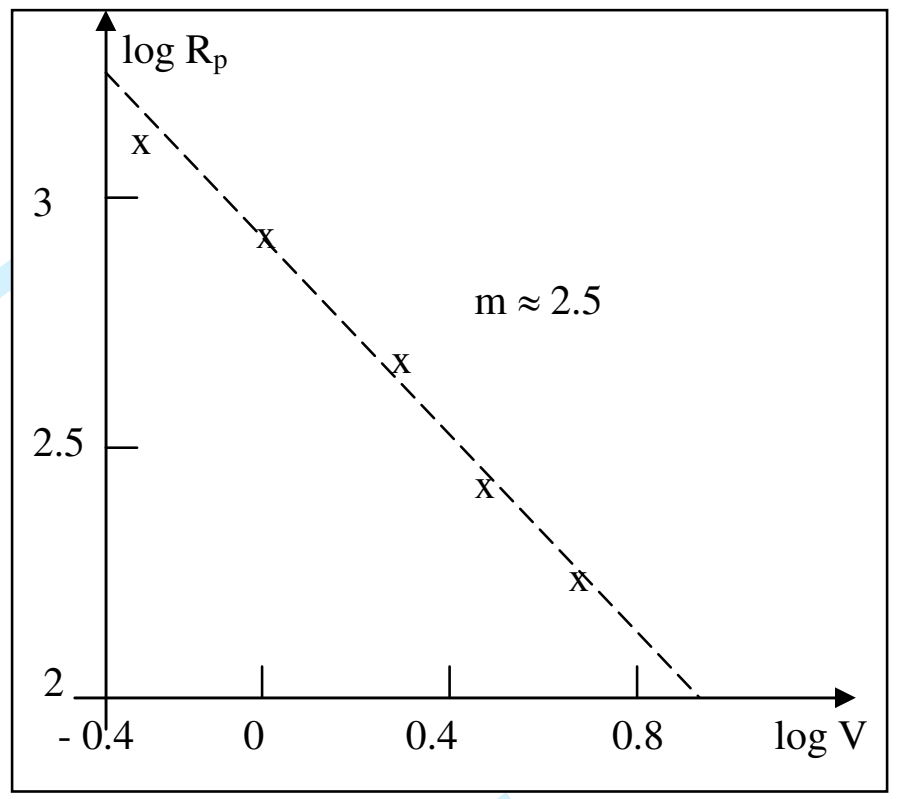

